\title{
Im Rucksack Motivation
}

\section{Internationale Freiwilligendienste als Katalysator für späteres Engagement in Deutschland}

\author{
JÖRN FISCHER UND \\ BENJAMIN HAAS \\ Dr. Jörn Fischer hat in den Jahren \\ 1996/97 den »Anderen Dienst im \\ Ausland " (Zivildienstersatz) in Mon- \\ tevideo/Uruguay geleistet. Im Jahr \\ 2005 Studienabschluss als Diplom- \\ Regionalwissenschaftler Lateiname- \\ rika an der Universität zu Köln; 2004 \\ bis 2008 Mitarbeiter und seit 2008 \\ assoziierter Wissenschaftler am \\ Lehrstuhl für Vergleichende Politik- \\ wissenschaft an der Universität zu \\ Köln. 2011 Promotion zum Dr. rer.pol. \\ joern.fischer@uni-koeln.de
}

Benjamin Haas hat im Jahr 2005 einen Freiwilligendienst in einem Kulturzentrum in Granada/Nicaragua geleistet sowie den "Anderen Dienst im Ausland «. Im Jahr 2011 Abschluss des Bachelor of Arts in Regionalstudien Lateinamerika an der Universität zu Köln. Von 2009 bis 2011 tätig am Seminar für Sozialpolitik an der Wirtschafts- und Sozialwissenschaftlichen Fakultät als verantwortlicher Redaktionsassistent der »Zeitschrift für öffentliche und gemeinwirtschaftliche Unternehmen«.

redaktion-zoegu@uni-koeln.de

\author{
Junge Menschen, die sich in einem internationalen \\ Dienst freiwillig engagieren, tun das zumeist \\ auch nach ihrer Rückkehr in Deutschland.
}

»Ein Freiwilligendienst endet nicht mit dem letzten Tag im Ausland «, heißt es in der von Absolventen eines internationalen Freiwilligendienstes verfassten »Potsdamer Erklärung «. (1)

Warum das? Weil viele zurückgekehrte Freiwillige aus den Erfahrungen des Dienstes eine Motivation schöpfen, sich auch nach dem Freiwilligendienst weiter zu engagieren. Allein über das entwicklungspolitische Förderprogramm »weltwärts « (2) haben in den vergangenen fünf Jahren über 10.000 junge Menschen aus Deutschland einen internationalen Freiwilligendienst geleistet.

Die Engagement-Bereitschaft dieser Rückkehrerinnen und Rückkehrer in der eigenen Gesellschaft ist überdurchschnittlich hoch und spielt eine immer größere Bedeutung im entwicklungspolitischen und im sozial-karitativen Bereich des Dritten Sektors in Deutschland. Wie lässt sich dieses besondere Phänomen erklären? Welche Formen des Rückkehr-Engagements lassen sich unterscheiden und welche empirischen Erkenntnisse gibt es dazu? Darauf gibt der vorliegende Beitrag Antworten.

\section{Ausgangspunkt und Definition}

Internationalen Freiwilligendiensten kommt heute nicht nur im Lebensentwurf immer mehr junger Menschen eine große Bedeutung zu. Sie sind auch in den Fokus der Engagement-Politik in Deutschland gerückt. Die aktuelle Bundesregierung beispielsweise hat die Förderung internationaler Freiwilligendienste in ihrer nationalen EngagementStrategie verankert (BMI 2010: 27 ff.).

Erst durch die Ausweitung des Freiwilligen Sozialen Jahres auf das Ausland und durch die Einführung des von der
Europäischen Union getragenen Europäischen Freiwilligendienstes (EFD) in den 1990er-Jahren können sogenannte "geregelte Freiwilligendienste « auch außerhalb Deutschlands geleistet werden (Stern u. a. 2011a:5).

Seither sind weitere öffentlich geförderte und mehr oder weniger staatlich geregelte Dienstformen entstanden, beispielsweise »weltwärts« (3), »kulturweit « (4) oder der Internationale Jugendfreiwilligendienst (5). Daneben gibt es konfessionell organisierte Freiwilligendienste wie das Diakonische Jahr im Ausland (6) oder Missionar auf Zeit (7) (Christ/Fischer 2012: 30 f). Darüber hinaus existieren eine Reihe sogenannter ungeregelter Dienstformen.

In den grundlegenden Charakteristika unterscheiden sich internationale Freiwilligendienste - abgesehen vom Ort der Erbringung - nicht von den nationalen Diensten. Sie sind eine besondere Form bürgerschaftlichen Engagements, gemeinwohl- und non-profit-orientiert, finden im öffentlichen Raum statt und dauern in der Regel von mehreren Monaten bis hin zu einem oder zwei Jahren (Guggenberger 2000: 636). Sie können daher wie folgt definiert werden:

»Bei einem internationalen Freiwilligendienst engagieren sich zumeist junge Menschen über einen (öffentlichen) Träger, der sie pädagogisch begleitet, für einen bestimmten Zeitraum freiwillig, aber verbindlich und ohne Absichten eines persönlichen materiellen Gewinns unter formalgeregelten Rahmenbedingungen in einem gemeinwohl- und NonProfit orientierten Betätigungsfeld im öffentlichen Raum einer Gesellschaft im Ausland.«(Haas 2012: 20).

Die Besonderheit eines internationalen Freiwilligendienstes ist also, dass er nicht im Heimatland der Freiwilligen 
stattfindet. Was zunächst banal klingt, hat jedoch eine weitreichende Bedeutung: Den Prinzipien der Gemeinwohlorientierung und des Stattfindens im öffentlichen Raum liegt bei internationalen Freiwilligendiensten eine Dualität zugrunde. Während das nachgelagerte Engagement der Freiwilligen auf das Gemeinwohl der eigenen Gesellschaft ausgerichtet sein kann, so steht das Engagement während des Einsatzes im Ausland in einem größeren Zusammenhang (Haas 2012: 19).

Die Konzepte der Global Citizenship oder des Kosmopolitismus bieten dafür Erklärungsansätze. Sie verorten handelnde Personen und Gruppen in einer globalisierten Welt in diesem größeren Zusammenhang (z. B. Köhler 2006). Ein gutes Beispiel ist der Europäische Freiwilligendienst, der von einer europäischen Gesellschaft ausgeht, die durch den Dienst gestärkt werden soll (Europäische Kommission 2011: 52 ff.). Der Geograf und Arbeitssoziologe Andrew Jones hat diese Annahme auch theoretisch begründet. Er sieht internationalen Freiwilligendienst grundsätzlich »as part of a developing global civil society« (Jones 2011: 542).

Und, wie später noch deutlich werden wird, zeigt sich empirisch die Besonderheit, dass gerade internationale Freiwilligendienste als Katalysator für späteres Engagement in der eigenen Gesellschaft fungieren. Das "weltwärts«Förderprogramm des Bundesministeriums für wirtschaftliche Zusammenarbeit und Entwicklung hat dies fest in seiner Zieldimension verankert. Durch das Rückkehrengagement der Freiwilligen soll ein »deutliche[r] Impuls für die entwicklungspolitische Inlands- und Bildungsarbeit in Deutschland im Sinne des >Globalen Lernens« (Stern u. a. 2011a: 7) gegeben werden.

\section{Dritter Sektor}

Internationale Freiwillige sind grundsätzlich im Dritten Sektor verortet. Wir folgen der Definition des Dritten Sektors von Schulz-Nieswandt und Köstler (2011:87), die jegliche Form bürgerschaftlichen Engagements dem Dritten Sektor zuordnen. Dieser wird innerhalb einer Vier-Felder-Matrix (8) als vielfältig und amorph analysiert (Schulz-Nieswandt/Köstler 2009: 32). Der Dritte Sektor wird dabei intermedi- är zwischen Staat, Markt und Familie/ Verwandtschaft konstruiert (SchulzNieswandt/Köstler 2011: 91).

Auf seine Ausgestaltung und die Rolle des bürgerschaftlichen Engagements innerhalb des Sektors (z. B. in der Wohlfahrtsproduktion) soll an dieser Stelle nicht näher eingegangen werden. (9) Wichtig ist, dass nach diesem Verständnis, das Engagement internationaler Freiwilliger im Ausland vom Dritten Sektor ausgeht, der zugleich das Handlungsfeld für das RückkehrEngagement der Freiwilligen darstellt.

\section{Theoretischer Ansatz: Reziprozität}

Aufopfernde Weltverbesserer oder karriereorientierte Nutzenmaximierer, die den eigenen Lebenslauf aufbessern wollen? In diesem Spannungsfeld wird in der Diskussion um internationale Freiwilligendienste sehr häufig die Motivation der Freiwilligen verortet. Damit ist diese der klassischen Dichotomie aus Altruismus versus Egoismus unterworfen (Haas 2012: 26 f.) - eine oberflächliche und einengende Perspektive auf internationalen Freiwilligendienst.

Die Reziprozitäts-Theorie hingegen bietet einen Ansatzpunkt, um das Phänomen internationaler Freiwilligendienste ganzheitlich zu verstehen. (10) Die Sozialtheorie um Gabe und Reziprozität geht auf den französischen Soziologen und Ethnologen Marcel Maus (1968, zuerst 1928) zurück. Seine Grundannahme ist, dass durch Geben, Nehmen und Erwidern soziale Beziehungen entstehen (Mauss 1968: 27 ff.).

Für die Erklärung bürgerschaftlichen Engagements und insbesondere internationaler Freiwilligendienste müssen wir die Reziprozität in der Form der »generalisierten Norm« als Erklärung heranziehen (Schulz-Nieswandt/Köstler 2011: 150). Im Gegensatz zur ausgeglichenen, direkten Reziprozität, wie sie etwa beim Markttausch vorliegt, kann bei der generalisierten Form Geben und Nehmen zeitlich auseinanderfallen.

Soziologisch ist jede Gabe immer an eine wie auch immer gelagerte Gegengabe geknüpft. Die generalisierte Reziprozität bildet aber eine Norm, die dafür sorgt, »dass auch dann in ein Geben eingewilligt wird, wenn es gar nicht beabsichtigt ist, das Geben mit dem Nehmen aufzurechnen « (SchulzNieswandt/Köstler 2011: 150). Cha- rakteristisch für die generalisierte $\mathrm{Re}$ ziprozität ist ein Gabeüberschuss, dem eingewilligt wird, ohne zu wissen, wie und ob eine Gegengabe erfolgen wird. Über den Zeitverlauf und die Akteure hinweg generalisierte Formen der Reziprozität sind in den Logiken internationaler Freiwilligendienste tief verankert und konstituieren unterschiedliche Beziehungen zwischen den verschiedenen Akteuren (Haas 2012: 82).

Konkret bedeutet dies: Freiwillige sind dazu bereit, sich für andere $\mathrm{zu}$ engagieren, ohne zu wissen, was sie dafür erhalten werden. Sie können Erwartungen haben durch die Erzählungen von ehemaligen Freiwilligen oder durch Programmversprechen, aber eine Gewissheit haben sie nicht. Sie bringen ein hohes Vertrauen auf und sind unbeachtet der individuellen Motive dazu bereit, einen relativen (11) bedingungslosen Gabeüberschuss zu leisten.

Während des Dienstes verschmelzen in ihrer Person Gaben und Gegengaben. Denn einerseits engagieren sie sich, setzen sich in den Einrichtungen ein, auf der anderen Seite lernen sie viel und nehmen einiges für ihr späteres Leben mit, was zahlreiche Studien belegen (Haßinger 2000; Mundorf 2000; Baldas 2003; Schüll 2004; BMFSFJ 2005).

Empirisch zeigt sich, dass viele Freiwillige das Gefühl haben, »mehr zu Lernen als zu Bewirken « (Schwinge 2011: 127). Das Rückkehr-Engagement der Freiwilligen kann somit verstanden werden, als die zeitlich nachgelagerte Gegengabe. Der Empfänger ist dabei nicht zwangsläufig die Einrichtung (oder die Menschen in der Einrichtung), die ihnen den Dienst ermöglicht hat, sondern kann durch die Generalisierung der Reziprozität eine Wärmestube für $\mathrm{Ob}$ dachlose in der eigenen Stadt oder die Gründung eines eigenen Vereins sein, der sich für entwicklungspolitische Bildung einsetzt.

Da es sich auch bei diesem Engagement wiederum gleichzeitig um Lernund Erfahrungsorte handelt, von denen die freiwillig Engagierten etwas bekommen, geht die Verkettung von Gaben und Gegengaben zu Reziprozitätsbeziehungen immer weiter. Hier wird deutlich, dass die Reziprozitätstheorie auch auf andere Bereiche bürgerschaftlichen Engagements anwendbar ist.

Das Rückkehr-Engagement der internationalen Freiwilligen ist äußerst divers 
in Inhalt und Struktur (Christ/Fischer 2012: 167 ff.). Die eigene Entsendeorganisation ist dabei häufig der erste Anlaufpunkt, doch trägerübergreifende Freiwilligenvereinigungen, andere Nicht-Regierungs-Organisationen mit regionalem oder inhaltlichem Bezug zum eigenen Dienst sind dabei als Engagement-Plattform genauso denkbar wie ein loser Zusammenschluss von Ehemaligen, der sich nur zur Planung und Umsetzung eines bestimmten Projektes zusammenfindet. Inhaltlich ist zumeist ein Bezug zum eigenen Dienst erkennbar, zumindest im weiteren Sinne: Wer beispielsweise in seinem Gastland mit den Folgen globaler Ungleichverteilung von Reichtum konfrontiert wurde, mag dies als Anstoß für ein Engagement in der entwicklungspolitischen Bildungsarbeit sehen.

Die wissenschaftliche Befassung mit den Auswirkungen von internationalen Freiwilligendiensten auf das spätere Engagementverhalten steht in Deutschland noch sehr am Anfang. Dies ist auch den methodischen Schwierigkeiten geschuldet, das Engagement ursächlich dem Freiwilligendienst zuzuschreiben. Wer sich vor dem Dienst engagiert hat, tut dies womöglich auch danach. Außerdem mag sich so manche Wirkung vielleicht erst Jahre nach dem Dienst zeigen und zu Engagement führen. Ungeachtet dieser forschungspraktischen Herausforderungen gibt es dennoch einige Erkenntnisse, die zahlreiche Ausstrahlungseffekte internationaler Dienste aufzeigen und deren Einflussfaktoren analysieren. Besonders umfassend und gleichzeitig methodisch elaboriert ist die Evaluierung des weltwärts-Freiwilligendienstes (Stern u. a. 2011a).

Grundsätzlich scheint der Einfluss eines internationalen Dienstes auf gesellschaftliches Engagement etwas größer zu sein als von Inlandsdiensten (Rahrbach et. al. 1998: 284). Die Gesamtheit der Diensterfahrung im Ausland, so eine mögliche Hypothese, ist ein wirkungsvollerer Katalysator als die Inlandserfahrung. Allerdings: Aus wissenschaftlicher Perspektive ist dieser Katalysator noch eine "Black Box", die durch weitere Forschung über den Motivationsquell für das Engagement danach noch erhellt werden muss.

Für "weltwärts« zeigt sich, dass sich 64 Prozent der Freiwilligen nach ihrer Rückkehr in Deutschland entwe- der allgemeingesellschaftlich oder entwicklungspolitisch engagieren (Stern u. a. 2011b: 23). Diese Zahl steigt, je länger die Freiwilligen zurück sind, was anhand eines approximierten Längsschnitts (12) gezeigt werden konnte. Bei der Gruppe, die schon länger als 14 Monate zurück in Deutschland war, engagierten sich bereits 69 Prozent.

Im Europäischen Freiwilligendienst sind bei etwa 50 Prozent der befragten Ex-Freiwilligen Engagement-Wirkungen vorhanden, die durch diesen Dienst induziert wurden (Becker et. al. 2000: 48). Dabei geht es um konkrete Handlungen und nicht etwa um Einstellungsänderungen oder bloße Engagementintention. Inhaltlich dominiert dabei der soziale Bereich, gefolgt von interkulturellem und politischem Engagement.

Die »weltwärts«-Evaluation wiederum zeigt, dass die Engagement-Quote offensichtlich steigt, wenn sich für die Freiwilligen die neue Lebensphase (Umzug in eine neue Stadt etc.) eingependelt hat. Von denen, die sich zum Zeitpunkt der Befragung nicht engagierten, gaben 68 Prozent an, sich "wahrscheinlich oder mit großer Sicherheit zukünftig freiwillig zu engagieren « (Stern u. a. 2011a: 24). Freiwillige, die als Motivation angeben, Gutes tun zu wollen oder sich persönlich entwickeln zu wollen, engagierten sich mit höherer Wahrscheinlichkeit (Stern u. a. 2011a: 46).

Die Engagement-Formen der »weltwärts «-Freiwilligen in Bereichen die nicht zur Entwicklungszusammenarbeit zählen (also im allgemeingesellschaftlichen und sozial-karitativen Bereich liegen) sind dabei sehr vielfältig und reichen von einer Mitgliedschaft in einem gemeinnützigen Verein (58 Prozent) sowie der Teilnahme an Veranstaltungen (57 Prozent) bis hin zu Initiierung von eigenen Projekten (24 Prozent) und Mitarbeit in Städtepartnerschaften (6 Prozent). 38 Prozent der Freiwilligen unterstützen ihre Entsendeorganisationen in der fachlich-pädagogischen Begleitung der zukünftigen Freiwilligengenerationen (Stern u. a. 2011b: 26). Offensichtlich ist es vielen ein Anliegen, das Erlebte weiterzugeben und bei der Vorbereitung der neuen Freiwilligen mitzuwirken.

Im Europäischen Freiwilligendienst zeigt sich, dass das Engagement überwiegend im Alltag und ehrenamtlich er- folgt. Die Bereitschaft der Freiwilligen, sich in bestehenden Organisationen zu engagieren, ist dabei gering ausgeprägt (Becker et. al. 2000: 49) - ein stärker selbstbestimmtes, projektorientiertes Engagement ist für viele ehemalige Freiwillige offensichtlich attraktiver als das Ehrenamt in bestehenden Strukturen.

Was befördert das Engagement nach dem Dienst? Dazu liegen punktuelle Erkenntnisse vor. Zum einen spricht vieles dafür, dass es auf die persönliche Prädisposition der Freiwilligen ankommt. Anders ausgedrückt: Auf welchen Boden fällt die Saat "weltwärts «? Darüber hinaus ist bekannt, dass die Zufriedenheit mit dem Rückkehrseminar die Wahrscheinlichkeit erhöht, dass sich die jungen Menschen im Anschluss freiwillig und entwicklungspolitisch engagieren (Stern u. a. 2011a: 46).

Diese Erkenntnis macht die wichtige Rolle der Trägerorganisationen deutlich. Sie haben eine große Verantwortung und können durch gezielte Informationen und Qualifizierungen die Engagement-Bereitschaft, die die Freiwilligen aus ihrer Erfahrung im Ausland mitbringen, auffangen und befördern. Sie wirken sozusagen als Katalysator für den Katalysator.

\section{Anmerkungen}

(1) www.b-b-e.de/fileadmin/inhalte/ aktuelles/2009/09/nl18_potsdamer_erklaerung.pdf

(2) www.weltwaerts.de

(3) Der entwicklungspolitische Freiwilligendienst des Bundesministeriums für wirtschaftliche Zusammenarbeit und Entwicklung (BMZ).

(4) Kultureller Freiwilligendienst des Auswärtigen Amtes in $\mathrm{Zu}$ sammenarbeit mit der Deutschen UNESCO-Kommission.

(5) Gefördert vom Bundesministerium für Familie, Senioren, Frauen und Jugend.

(6) Ein Programm der Jugendarbeit und Diakonie der Evangelischen Kirche in Deutschland.

(7) Wird von katholischen Missionsorden angeboten.

(8) Alle Sektoren werden dabei idealtypisch verstanden (Schulz-Nieswandt und Köstler 2011: 91).

(9) Siehe dazu umfassender die Darlegungen von Schulz-Nieswandt (2008). 
(10) Eine umfangreiche Anwendung auf den Kontext internationaler Freiwilligendienste erfährt dieses theoretische Konzept erstmals bei Haas 2012.

(11) Die Bedingungslosigkeit kann nur relativ sein, denn eine absolute kann es theoretisch nicht geben. Diese Erkenntnis ist philosophisch und theologisch begründet. Siehe dazu ausführlich Schulz-Nieswandt/Köstler 2011: $151 \mathrm{f}$.

(12) Da nur eine einzige Befragung durchgeführt wurde, konnten Veränderungen bei einzelnen Individuen über den Zeitverlauf nicht gemessen werden. Da die Zahl der Befragten jedoch so hoch war, konnten die Freiwilligen vor, in und nach ihrem Dienst miteinander verglichen werden. Die Rückgekehrten wurden nochmals in zwei Gruppen aufgeteilt, je nachdem, wie lange sie schon zurück waren. Damit lassen sich also Unterschiede zwischen den Gruppen zeigen und - in Evaluierungssprache - Veränderungen auf individueller Ebene »approximieren «.

\section{Literatur}

Becker, Roland/Brandes, Heike/Bunjes, Ulrike/ Wüstendörfer, Werner (2000): Lern- und

Bildungsprozesse im europäischen Freiwilligendienst. Band 1: Jugend für Europa - Deutsche Agentur Jugend, Bonn.

\section{Andrea Rahrbach/Werner Wüstendörfer/} Thomas Arnold (1998): Untersuchung zum Freiwilligen Sozialen Jahr, Stuttgart u. a. Baldas, Eugen (2003): Freiwilligendienste haben es in sich: Studien zu Art, Umfang und Ausbaumöglichkeiten von Freiwilligendiensten im kirchlich-sozialen Umfeld. Freiburg im Breisgau: Lambertus.

BMFSFJ (2005): Ergebnisse der Evaluation des FSJ und FÖJ, Abschlussbericht des Instituts für Sozialforschung und Gesellschaftspolitik e. V. Köln und Berlin. Köln-Berlin.

BMI (2010): Nationale Engagementstrategie der Bundesregierung. Abgerufen am 14. April 2012 (www.bmi.bund.de/SharedDocs/Downloads/DE/Themen/Politik_Gesellschaft/ GeselZusammenhalt/engagementstrategie. pdf?_blob=publicationFile).

Christ, Katja/Jörn Fischer (2012): Internationale Freiwilligendienste: Lernen und Helfen im Ausland, vierte Auflage. Freiburg im Breisgau: Interconnections.
Europäische Kommission (2011): Youth in Action Programme Guide. Abgerufen Juli 22, 2011 (www.action2.eu/int/youth-in-action/guide20 11/87F3F73A85752542BC38A1E868B5E2AD/).

Guggenberger, Bernd (2000): Jugend erneuert Gemeinschaft: Freiwilligendienste in Deutschland und Europa. Eine Synopse. Baden-Baden: Nomos.

Haas, Benjamin. 2012. Ambivalenz der Gegenseitigkeit. Reziprozitätsformen des weltwärtsFreiwilligendienstes im Spiegel der PostkoIonialen Theorie. Interdisziplinäre Studien zu Freiwilligendiensten. Band 2. Herausgegeben von Jörn Fischer. Köln: Kölner Wissenschaftsverlag.

Haßinger, Kerstin. 2000. Unausweichliche Nähe: eine explorative Studie zu den Erfahrungen Freiwilliger in internationalen Diensten und den Auswirkungen der Erfahrungen nach der Rückkehr in die Heimat. Freiburg im Breisgau.

Jones, Andrew. 2011. Theorising international youth volunteering: training for global (corporate) work? Transactions of the Institute of British Geographers (36):530-544

Köhler, Benedikt. 2006. Soziologie des Neuen Kosmopolitismus. Wiesbaden: VS Verlag für Sozialwissenschaften.

Mauss, Marcel. 1968. Die Gabe: Form und Funktion des Austauschs in archaischen Gesellschaften. Frankfurt am Main: Suhrkamp Verlag. Mundorf, Margret. 2000. Christliche Freiwilligendienste im Ausland. Lernprozesse und Auswirkungen auf die Lebensentwürfe junger Menschen. Eine qualitative Studie. Münster [u. a.]: Waxmann.

Schüll, Peter. 2004. Motive Ehrenamtlicher: eine soziologische Studie zum freiwilligen Engagement in ausgewählten Ehrenamtsbereichen. Berlin: Wissenschaftlicher Verlag Berlin. Schulz-Nieswandt, Frank. 2008. Zur Morphologie des Dritten Sektors im Gefüge zwischen Staat, Markt und Familie. Ein Diskussionsbeitrag zur Ciriec-Studie »Die Sozialwirtschaft der Europäischen Union«. Zeitschrift für öffentliche und gemeinwirtschaftliche Unternehmen (31(3)): 323-336.

Schulz-Nieswandt, Frank, und Ursula Köstler. 2009. Bürgerschaftliches Engagement: Grundlagen und Perspektiven. S. 29-41 in Niedrigschwellige Betreuung von Demenzkranken: Grundlagen und Unterrichtsmaterialien, herausgegeben von Gabriela Stoppe und Gerthild Stiens. Stuttgart: Kohlhammer.

Schulz-Nieswandt, Frank, und Ursula Köstler. 2011. Bürgerschaftliches Engagement im Alter. Hintergründe, Formen, Umfang und Funktionen. Stuttgart: Kohlhammer.

Schwinge, Brigitte. 2011. Verkehrte Welten: Über die Umkehrung der Verhältnisse von
Geben und Nehmen. Der weltwärts-Freiwilligendienst als Selbstbehandlung im Kulturkontakt zwischen Deutschland und Südafrika. Interdisziplinäre Studien zu Freiwilligendiens ten. Band 1. Herausgegeben von Jörn Fischer. Bonn: Scientia Bonnensis.

Stern u. a. 2011a. Der entwicklungspolitische Freiwilligendienst »weltwärts«. Band I: Hauptbericht. Unveröffentlichter Evaluierungsbericht. Bonn: Bundesministerium für wirtschaftliche Zusammenarbeit und Entwicklung. Stern u. a. 2011b. Der entwicklungspolitische Freiwilligendienst »weltwärts». Band III: Entwicklungspolitische Inlands- und Bildungsarbeit durch die Rückkehrarbeit von »weltwärts«-Freiwilligen. Unveröffentlichter Evaluierungsbericht. Bonn: Bundesministerium für wirtschaftliche Zusammenarbeit und Entwicklung

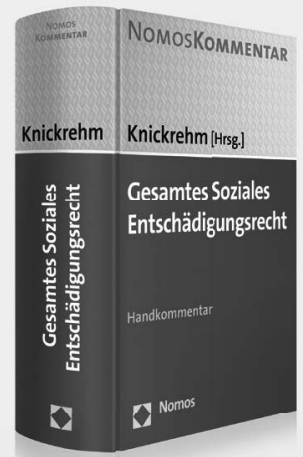

\section{Gesamtes Soziales Entschädigungsrecht}

Handkommentar

Herausgegeben von RiBSG Sabine Knickrehm 2012, 1.080 S., geb., 98,-€ ISBN 978-3-8329-5275-4

Der aktuelle Handkommentar bietet grundlegende, neue Systematisierungs- und Interpretationsansätze und enthält paragrafengenaue Kommentierungen der wichtigsten Regelungen vom BVG bis zum ZDG.

www.nomos-shop.de/12158

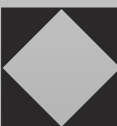

Nomos 